\title{
THE FEATURES OF CENTRAL NERVOUS SYSTEM DAMAGE IN CHILDREN WITH CONGENITAL CYTOMEGALOVIRUS INFECTION
}

\author{
Gonchar M., Urivaeva M., Muratov G., Malich T., Omelchenko H., \\ Shapko M., Liesna A., Poliakova V. \\ Kharkiv National Medical University, Ukraine \\ Kharkiv Regional Children Hospital \\ https://doi.org/10.35339/ic.7.2.89-93
}

\begin{abstract}
The paper discusses the features of central nervous system lesions in children with congenital cytomegalovirus infection at different stages of gestation. In the course of the work, an algorithm for diagnosing congenital cytomegalovirus infection was developed. To demonstrate the complexity of diagnosis and treatment in a child of the first year of life, a clinical observation is given. The positive dynamics in the treatment of children with the use of human anticytomegalovirus immunoglobulin with a high content of IgG is discussed.

Key words: damage, central nervous system, congenital, young children, anticytomegalovirus.
\end{abstract}

\section{Introduction}

For several decades, herpes virus infection has remained an urgent multidisciplinary problem in pediatrics, infectology, obstetrics and gynecology. According to the World Health Organization (2018), a pandemic of herpes virus infections is observed: $90 \%$ of adults and children on the planet has been infected with herpes viruses, and 50\% of them have a manifest, recurrent course [1].

Human cytomegalovirus (CMV) is a $\beta$-herpesvirus that can cause a primary infection. For the first time in the laboratory, the virus was cultivated by M. Smith (1955). Infection of infants with CMV occurs with breast milk in 57\% [Kulikov VI, 2017], through saliva and blood transfusions, during transplantation of internal organs and hematopoietic stem cells, through the affected genital tract of the mother in $63 \%$ [Karazhas NV , 2015]. According to the American Public Health Center, of 4 million births per year in the United States, about $1 \%$ of children with congenital CMV infection are born; 8700 children annually have early and late complications of CMV infection [Frize K. 2013]. According to

Corresponding Author:

Maryna Urivaeva, MD, PhD, Associate Professor,

Department of Pediatrics No. 1 and Neonatology,

Kharkiv National Medical University,

E-mail:marina310366@gmail.com
Walker S. P. (2013), 3.7-20\% of pregnant women in the United States were allocated CMV in the material taken from the cervical canal. The incidence peak encompasses two time intervals: up to three years $-39 \%$ of patients; $16-25$ years $41 \%$. [2] In the study of CMV infection in pregnant women, primary CMV infection was found in $32.3 \%$ of women with the risk of termination of pregnancy, in $18.1 \%$ - spontaneous abortion, in $39.5 \%$ - premature birth, in $54.4 \%$ with signs of polyhydramnios, $35,2 \%$ - with a dead pregnancy [Revello M. G 2015]. After infection, $\mathrm{CMV}$ is often transformed into a latent form with periodic reactivation in the host organism [3]. In immunocompetent people, the infection is asymptomatic, and in some patients has clinical manifestations of infectious mononucleosis, splenomegaly is diagnosed. Despite the fact that $\mathrm{CMV}$ is the main infectious cause of sensorineural hearing loss and abnormalities of the development of the central nervous system (CNS), congenital CMV infection is poorly diagnosed and is detected only in $0.2-2.0 \%$ of pregnant women [4-6] .

\section{Purpose, subjects and methods:}

2.1. The purpose of the study was to analyze modern medical literature on CNS disorders in children with congenital CMV infection.

\subsection{Subjects \& Methods}

The basis of the study is the study of open scientometric and abstract databases PubMed, 
HINARI, Google Scholar using bibliographic and analytical-syntactic methods.

\section{Conflict of interests.}

There is no conflict of interests

\section{Results \& Discussion}

Informal International Congenital Cytomegalovirus Recommendations Group (ICCRG) congenital CMV infection group identified major risk factors for disease (2015), including: lack of maternal or newborn screening programs, low level of routine testing of infants who are at risk and parents about infections during pregnancy, limited efficacy and high toxicity of modern therapies during pregnancy, the absence of licensed vaccines [7-9].

CMV infection is a viral disease characterized by polymorphic clinical symptoms resulting from the formation in the salivary glands, visceral organs and the CNS of specific cytomegal cells.

The International Classification of Diseases (ICD-10), distinguished the following CMV infections:

P35.1 Congenital CMV infection

B25 Cytomegalovirus disease

B25.0 Cytomegalovirus pneumonitis

B25.1 Cytomegalovirus hepatitis

B25.2 Cytomegalovirus pancreatitis

B25.8 Other cytomegalovirus diseases

B25.9 Cytomegalovirus disease, unspecified

In clinical practice, the following classification of CMV infection is used:

1) by nature of infection: congenital, acquired;

2 ) by the course: acute (up to 3 months), subacute (3-6 months), prolonged (6-12 months), chronic (more than 12 months);

3 ) in the form: localized, distributed, mixed, generalized;

4) by stages of the disease: active, manifestations, relapse with activation period, active stage, stage of convalescence, stage of compensation.

CMV can be transplanted translucently when a woman develops a primary CMV infection during pregnancy or as a result of latent reactivation of the virus acquired prior to pregnancy. [10]. The risk of transmission of fetal infection is higher among pregnant women with primary infection compared to those who were IgGpositive for pregnancy. Vertical transmission of CMV fetal infection is more common as a result of maternal infection in the third trimester of pregnancy, but the incidence of CNS complications is lower among children infected in the third trimester than infants born to women with primary CMV infection in the first trimester $[11,12]$.
Depending on the gestation period, CMV infection is manifested by different clinical symptoms. When infected in the period from 16 to 75 days, embryopathies develop, because CMV exhibits high neurotropism, almost $60 \%$ of cases of manifestations of embryopathy are organic CNS lesions in the form of: hydrocephalus $24 \%$; microcephaly $-15 \%$, ventriculomegaly $11 \%$, hypoplasia of brain structures $-6 \%$, delay of myelination $-4 \%$, and others [13]. The disorders of the cardiovascular system, in the form of defects in the development of the valvular apparatus of the heart, defects of the atrial and interventricular membranes, are equally important. Infection within the period from 76 to 180 days results in development of fetopathies, which are manifested by fibro-sclerotic deformities of the organs $-15 \%$, miscarriage $-5 \%$, hepatitis $-23 \%$, encephalitis $-11 \%$, paraventricular cysts $-4 \%$, calcification of the substance of the brain \%, chorioretinitis $-7 \%$, neurosensory hearing loss $27 \%$, pneumonia $-5 \%$ [14].

The nature and timing of the manifestation of congenital CMV infection depends on the premorbid condition of the newborn, namely, the burden of obstetric history, immaturity, the presence of hereditary diseases, concomitant pathology, in weak, prematurely born children, manifestation of infection can be observed 4-5.

The first clinical signs of congenital CMV in newborns are manifestations of CNS lesions in the form of: suppression of unconditional swallowing reflexes, suction $-21 \%$, focal symptoms and manifestation of strabismus $-16.3 \%$, nystagmus $27 \%$, asymmetry of facial muscles $-6 \%$, muscle tone in the form of: hypertonicity $-13.1 \%$, hypotension $-29.8 \%$, or mixed form $-10.1 \%$, in the presence of hydrocephalus, there are signs of increased intracranial pressure, namely: a significant increase in the circumference of the baby's head in a short time $-13,2 \%$, blasting of the thigh $-22,9 \%$, with "fountain" regurgitation $24.8 \%$, convulsive syndrome $-7 \%$.

According to Grosjean J. (2014), children with CNS lesions showed signs of encephalitis (31.2\% of cases), which manifested from the second week of life and had a wavy course with early in $14.1 \%$ and late in $-23.4 \%$ exacerbations. Children with encephalitis had manifestations of intoxication, impaired consciousness in the form of inhibition (27.4\%), as well as resistance (19.6\%). Focal symptoms in the form of horizontal nystagmus $-35.4 \%$, strabismus $-8.2 \%$, vomiting $12.3 \%$, convulsive syndrome $-17 \%$ appear approximately one week after the onset of the 
disease, requiring additional vigilance for the diagnosis of encephalitis in children with congenital CMV infection.

In the majority of children (up to $85 \%$ ), congenital CMV infection occurs in subclinical form. In $35 \%$ of these children manifestation of congenital infection with CNS lesions is observed for 3-7 years of life and manifests neurosensory hearing loss $-41 \%$, visual impairment in the form of partial $-19,1 \%$, or complete atrophy of the optic nerve-24\%, convulsions $-23,5 \%$, pseudobulbar syndrome $-6,7 \%$, and episyndrome formation $11,7 \%$. There are also motor disorders by type of pyramidal insufficiency $-60.7 \%$, pediatric cerebral palsy $-23.1 \%$ of children, delay of speech and psychomotor development $-80.1 \%$, disorders of behavior $-5 \%$.

According to foreign literature, up to $12 \%$ of infants with clinical manifestations of CMV infections die, and most newborns who are infected early in pregnancy die within the first 10 days of life, and even in such a case it is difficult to identify the cause of death and verify the cause. According to the literature, $73.8 \%$ newborns with the generalized form of congenital CMV and 9.5\% with cerebral one tend to die [15]. Thus, the number of deaths in the first month of life is $37.1 \%$, at the age of 1-3 months $-18.3 \%, 4-6$ months $4.8 \%, 7-12$ months $-1.6 \%$. According to the pathoanatomical study, in $51.6 \%$ of the dead, there were signs of pre-natal infection in the form of: low body weight, encephalitis, meningitis, calcifications of the substance of the brain, hepatitis, many fetodysplasias, such as aortic hypoplasia, kidney, dysplasia valves, premature closure of fetal communications [16].

According to Prince HE (2014), the pathomorphological study of the placenta of patients with congenital CMV infection revealed subchoral intervals $-21.1 \%$, purulent widespread chorioamnionitis $-10.5 \%$, accelerated maturation of chorionic villi $-14.7 \%$ blood flow $-6.1 \%$, which led to fetal hypoxia and delayed prenatal development.

It is recommended to do the test on seroconversion, IgM antibody studies and IgG avidity index for CMV for the baby from the pregnancy with a flu-like symptoms (fever, severe fatigue, headache) that do not related to another established infection, and also for the fetus with a suspected CMV infection by the results of ultrasound examination [17].

To demonstrate the complexity of diagnosis and treatment in a child of the first year of life, we present the following clinical observation.
A 5-month- old patient was brought to the regional children's clinical hospital because of delayed psychomotor development. The baby was born from the first pregnancy (against smoking of the pregnant woman and infection with herpes virus infection with episodes of fever in the third trimester of pregnancy, delayed fetal fetal development), at 37 weeks, with a body weight of $2750 \mathrm{~g}$, body length $50 \mathrm{~cm}$.

Previously at the age of 2 months she was hospitalized because of fever to 39.1, mucous discharge from the nose, dry cough, delayed psychomotor development in the form of suppression of reflexes, muscle, inability to hold head, strabismus. The treatment resulted in improvement of the child's condition, the patient was discharged from the hospital.

At the age of 4 months, she was re-hospitalized at a regional children's clinical hospital because of fever, catarrhal manifestations from the upper respiratory tract. General condition of the child was severe due to fine intoxication. Psychomotor development did not match the age of the baby, there was jaundice of the skin, hepatosplenomegaly. Clinical blood test showed leukocytosis with a shift of the leukocyte formula to the left, accelerated ESR, monocytosis. Biochemical analysis of blood demonstrated five-fold increase in the liver tests (ALT, AST). Three-fold increase of anti-CMV IgM and IgG was revealed by enzyme immunoassay.

Congenital CMV infection with atypical hepatitis and developmental delay was diagnosed.

After the treatment, positive dynamics was noted and the patient was discharged from the hospital.

At the age of 5 months, she was admitted to the hospital due to delayed psychomotor development. Upon admission, the condition of the child was of moderate severity, the consciousness is clear. There were signs of delayed psychomotor development, namely, poorly expressed orientation in space, voice response was reduced, unconditioned reflexes were expressed weakly, there was muscular dystonia, inability to roll over, poor head retention, decreased reaction of sensorimotor behavior, squinting hepatosplenomegaly.

Clinical blood test showed $\mathrm{Hb}-92 \mathrm{~g} / \mathrm{l}, \mathrm{RBC}-$ $3,6 \times 1012 / 1$, blood quotient $-0,8, \mathrm{WBC}-9,5 \times$ $109 / 1$, eosinophils $-1 \%$, rod-core neutrophils $1 \%$, segmented neutrophils $-48 \%$, lymphocytes $40 \%$, monocytes $-10 \%$, the erythrocyte sedimentation rate was $15 \mathrm{~mm} / \mathrm{h}$. clinical urinalysis demonstrated the signs of leukocyturia (leukocytes in the entire field of vision). Biochemical blood test showed total protein $-62 \mathrm{~g} / \mathrm{l}$, alanine 
aminotransferase - 68 units/1, aspartate aminotransferase - 95 units/1, cholesterol - 3.8 $\mathrm{mmol} / \mathrm{l}$, the level of nitrogenous slag: creatinine $0.052 \mathrm{mmol} / 1$, urea $-2,9 \mathrm{mmol} / 1$. Enzyme-linked immunosorbent assay revealed anti-CMV IgM and IgG. PCR revealed the viral DNA in the blood.

To diagnose CMV infection we developed and used a diagnostic algorithm which is presented in Figure.
Specific anticytomegalovirus immunoglobulins are prescribed together with antiviral drugs. Detoxification therapy is performed depending on the degree of severity, if a mild and moderate degree prescribe copious drinking in the form of fruit, vegetable juices, then in severe condition requires the use of infusion therapy. Regarding the effectiveness of the treatment, there is a regression of clinical symptoms, according to

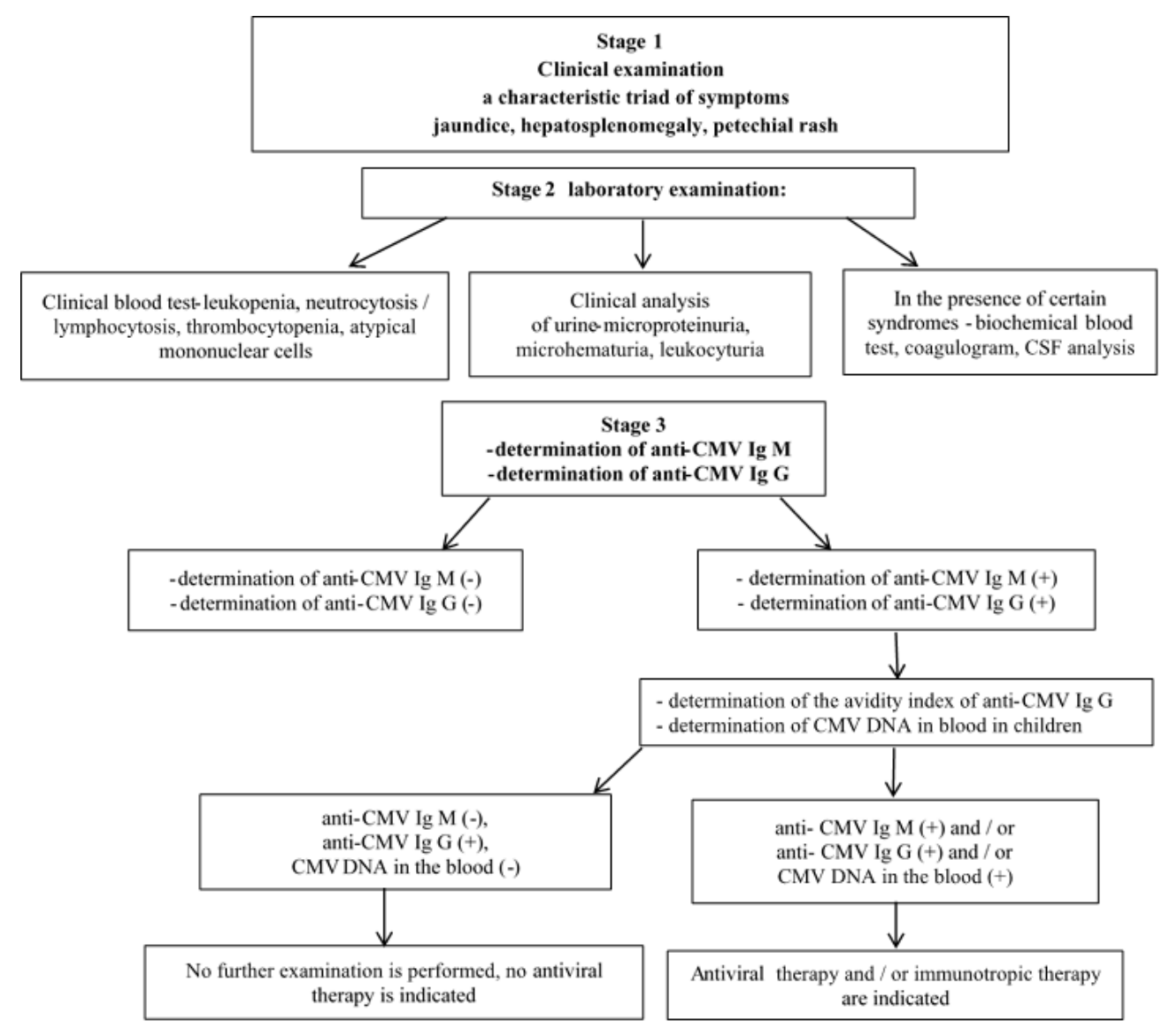

Diagnostic algorithm of CMV infection

In view of the foregoing, a clinical diagnosis the following diagnosis was made: "Congenital CMV infection. Atypical hepatitis. Anemia of mild severity. Uncomplicated pyelonephritis. Delay in psychomotor development".

Treatment of CMV infection consisted of etiotropic and symptomatic therapy. According to modern treatment protocols, etiotropic therapy involves administration of antiviral drugs: Ganciclovir (cymeven) 5-7.5 mg/kg / day in 2 doses with an interval of 12 hours, the course of treatment 1421 days; recombinant Interferon alfa-2 (Viferon) rectal suppositories of $150000 \mathrm{IU}$, or Foscarnet (a synthetic antiviral drug that suppresses DNA polymerase) is used as a second-line drug in the event of resistance to ganciclovir or due to side effects (level of evidence B). laboratory studies - the absence of virus DNA in the blood, the absence of anti-CMV Ig M and anti-CMV IgG with low avidity, the presence of anti - CMV IgG with high avidity [18].

Cytotect (Biotest Pharma GmbH, Germany), a human anticytomegalovirus immunoglobulin with high Ig G content (evidence level B) was administered. These antibodies are complementary to glycoproteins that are on the membrane of the virus. Neutralization of these glycoproteins makes it possible to prevent the damage to healthy cells and to reduce further virus replication.

The cytotect was administered as IV drops $2 \mathrm{ml} / \mathrm{kg}$ every 48 hours. The course of treatment of the child consisted of 5 injections. Dynamic observation after the first administration demonstrated a noticeable regression of neurological 
symptoms, normalization of the orientation in space, increased muscle tone, appearance of the ability to turn and hold the head. The patient was discharged on the $14^{\text {th }}$ day with positive dynamics. Screening for active CMV infection were was recommended on the month 1, 3, 6, 12 after the discharge from the hospital.

Conclusion. Congenital CMV infection is a poorly diagnosed infection, causing anomalies of the nervous system development. Important aspects are the prevention of the development of CMV in pregnant women, the prevention of its vertical transmission to the fetus, the early detection and treatment of the disease in the newborn.
Particular attention is given today to congenital CMV infection in newborns, which may clinically manifest or have an asymptomatic course. In this regard, it is recommended that all children with sensorineural hearing loss and intrauterine development should be screened for CMV infection (PCR diagnosis of saliva and urine).

Treatment options for congenital CMV infection are limited and include antiviral therapy and passive immunization with specific intravenous immunoglobulin. There are positive results that demonstrate the clinical efficacy of passive immunization with intravenous immunoglobulin containing specific IgG antibodies against the CMV pathogen.

\section{References}

1. Manicklal S., V.C. Emery, T. Lazzarotto, S.B. Boppana, R K.Gupta (2013). The "silent" global burden of congenital cytomegalovirus. Clin Microbiol Rev., 26, 86-102.

2. Hamilton S.T., W. van Zuylen, A. Shand. (2014) Prevention of congenital cytomegalovirus complications by maternal and neonatal treatments: a systematic review. Rev Med Virol., 24, 420-433.

3. Tey S.K. F. Goodrum, R.hanna. (2010). CD8+ T-cell recognition of human cytomegalovirus latency-associated determinant pUL138. J Gen Virol., 91, 2040-2048.

4. Doutre S.(2015).Reducing congenital cytomegalovirus infection through policy and legislation in the United States. Microbiology Australia., 11, 162-164

5. Walker S.P., R.Palma-Dias, E.M. Wood, P. Shekleton, M.L. Giles (2013). Cytomegalovirus in pregnancy: to screen or not to screen. BMC Pregnancy Childbirth, 13, 96.

6. Revello M.G., C. Tibaldi, G. Masuelli. (2015). Prevention of primary cytomegalovirus infection in pregnancy. Biomedicine, 2, 10

7. Wang C., X. Zhang, S.Bialek, M.Cannon. (2011). Attribution of congenital cytomegalovirus infection to primary versus non-primary maternal infection. J. Clin Infect Dis., 52, 11-13.

8. Munro S.C., B. Hall, L.R. Whybin. (2015). Diagnosis of and screening for cytomegalovirus infection in pregnant women. J Clin Microbiol., 44, 4713-4725.

9. Prince H.E., M. Lape-Nixon. (2014) Role of cytomegalovirus (CMV) IgG avidity testing in diagnosing primary CMV infection during pregnancy. Clin Vaccine Immunol., 21, 1377-1384.

10. Leruez-Ville M., J. Stirnemann, Y. Sellier. (2016). Feasibility of predicting the outcome of fetal infection with cytomegalovirus at the time of prenatal diagnosis. Am J Obstet Gynecol., 215, 1-9.

11. Arvin A. M. P. Fast, Myers, M. Plotkin. (2014). Vaccine development to prevent cytomegalovirus disease: report from the National Vaccine Advisory Committee. Clin Infect Dis., 39, 233-239.

12. Pass R. F., C. Zhang, A. Evans, T. Simpson. (2013). Vaccine prevention of maternal cytomegalovirus infection. N Engl J Med., 360, 1191-1199.

13. Bernstein D.I., F.M. Munoz, S.T. Callahan. (2016). Safety and efficacy of a cytomegalovirus glycoprotein B (gB) vaccine in adolescent girls: a randomized clinical trial. Vaccine., 34, 313-319.

14. Adler A.B. (2014). Cytomegalovirus and child day care: risk factors for maternal infection. Pediatr Infect Dis J., 11, 590-599.

15. Grosjean J., L. Trapes, S. Hantz. (2014). Human cytomegalovirus quantification in toddler's saliva from day care centers and emergency unit: a feasibility study. J. Clin. Virol., 61, 371-377.

16. Boppana S.B., S.A. Ross, M. Shimamura. (2011). Saliva polymerase-chain-reaction assay for cytomegalovirus screening in newborns. N. Engl. J. Med., 364, 2111-2118.

17. Revello M.G., C. Tibaldi, G. Masuelli. (2015). Prevention of primary cytomegalovirus infection in pregnancy. EbioMedicine, 2, 1205-1210.

18. Buxmann H., O.M. Stackelberg, R.L. Schlosser. (2011). Use of cytomegalovirus hyperimmunoglobulin for prevention of congenital cytomegalovirus disease: a retrospective analysis. J. Perinat Med., 40, 439-446. 